\title{
Modalités de régulation du processus de travail dans les activités de service en crèche
}

Regulation modalities of the working process in crèches

Modalidades de regulación del proceso de trabajo en Guardería

Rafael González et Annie Weill-Fassina

\section{CpenEdition}

\section{Journals}

Édition électronique

URL : http://journals.openedition.org/activites/1787

DOI : 10.4000/activites. 1787

ISSN : $1765-2723$

\section{Éditeur}

ARPACT - Association Recherches et Pratiques sur les ACTivités

\section{Référence électronique}

Rafael González et Annie Weill-Fassina, « Modalités de régulation du processus de travail dans les activités de service en crèche », Activités [En ligne], 2-2 I octobre 2005, mis en ligne le 01 octobre 2005, consulté le 10 décembre 2020. URL : http://journals.openedition.org/activites/1787 ; DOI : https:// doi.org/10.4000/activites. 1787

\section{$@ \oplus \Theta \Theta$}

Activités est mis à disposition selon les termes de la licence Creative Commons Attribution - Pas d'Utilisation Commerciale - Pas de Modification 4.0 International. 


\title{
Modalités de régulation du processus de travail dans les activités de service en crèche
}

\author{
Rafael González \\ Institut des Hautes Etudes en Santé "Dr. Arnoldo Gabaldon" \\ Ministère de la Santé et du Développement Social \\ C. P. : 4739, Zone Postale : 2101-A, Maracay - Venezuela \\ 8038ra@cantv.net / gonzal@cnam.fr
}

Annie Weill-Fassina

Laboratoire d'Ergonomie Physiologique et Cognitive, École Pratique des Hautes Études (EPHE) 41 rue Gay-Lussac, 75005 PARIS - FRANCE

Ephe.weill-fassina@wanadoo.fr

\begin{abstract}
Regulation modalities of the working process in crèches

The aim of this ergonomic research is to describe some regulation modalities of the process of decision and action that are used at different levels of work for managing service activities in crèches. We'll underline why and how executives and nurses adapt the preliminary rules resulting from various administrative authorities to try to ensure the quality of services for parents and children. Results are inferred from several methods, that is qualitative and quantitative, individual and collective analysis. The quality of service activities can be characterized by the interaction tuning between nurses and children. But this quality depends mainly upon the regulations that are done before in the main system decision centres.
\end{abstract}

KEYWORDS

Service activities, regulations, workers of child welfare, crèche.

\section{1.- Objectif : Comprendre la dynamique des activités de service en crèche}

L'objectif de cette recherche est de décrire les régulations du processus de décisions et d'actions qui interviennent, en France, dans les relations de service psycho-socio-éducatives en crèches, depuis leur conception institutionnelle jusqu'au travail des Professionnelles de la Petite Enfance en sections (PPE).

Ce travail est une «activité de service centrée sur autrui » (Letablier, 2001) puisque le rôle de ces personnels « est d'assurer aux enfants leur bien-être, de garantir leur sécurité, de répondre à leurs besoins et de les aider à se développer sans se substituer aux parents » (Décret n²000-762, rt. R. 180-1). Cette mission s'inscrit à un niveau institutionnel dans les cadres législatif et administratif du service public qui régissent le fonctionnement des crèches pour les enfants de 0 à 3 ans.

La demande à l'origine de cette recherche était de comprendre les causes d'un problème récurrent d'absence chez les personnels de crèche d'une collectivité locale pour améliorer les conditions de travail et la qualité de l'accueil des enfants. Ces absences étaient sources de conflits avec la Direction des Ressources Humaines de la collectivité locale dont dépendaient les crèches et les Directrices, la première les attribuant à de nombreux congés maladies et les secondes à la diminution de la durée hebdomadaire du travail en lien avec la loi sur les 35 heures. D'où des demandes réitérées 
d'effectifs supplémentaires soutenues par les parents en tant qu'usagers, demandes refusées puisque le taux légal d'encadrement était respecté. En fait, l'objet de l'intervention était double : éclairer le fonctionnement des crèches et identifier les fonctions présentes, les enjeux et les rôles respectifs des différents personnels. D'où une recherche sur les activités des Professionnelles de la Petite Enfance en crèche qui a intégré le problème critique des absences dans son contexte pour en comprendre les sources et les effets.

\section{2.- Intérêt social : Spécifier les caractéristiques des activités de service en crèche}

L'intérêt social de cette recherche est de préciser et de faire reconnaître les spécificités des métiers de la petite enfance en relation avec leurs conditions d'exécution et, par suite, leur hétérogénéité par rapport aux relations administratives et commerciales (Grosjean, 2000 ; Gonzales, Claire-Louisor, \& Weill-Fassina, 2001). Plusieurs catégories de personnels interviennent auprès des tout-petits, avec des attributions différentes : au niveau managérial, les puéricultrices et les infirmières font le plus souvent fonction de cadres de direction ; au niveau de la réalisation, les auxiliaires de puériculture et les éducatrices interviennent en section. Le métier est largement féminisé dans la mesure où les tâches qu'elles effectuent sont considérées comme « suppléances domestiques » (Durning, 1986), ce qui génère un manque de reconnaissance (Jenson, \& Sineau, 2001). Mais les définitions des missions sont peu explicites quant aux moyens, techniques, savoir-faire et compétences requis.

Ce type de relation de service est relativement peu étudié probablement pour ces raisons de proximité avec la vie familiale et domestique et aussi pour des raisons de difficultés d'approche méthodologique étant données les dimensions éducatives, affectives et domestiques qui s'y trouvent entremêlées. La plupart des études et recherches ont été moins orientées vers le travail des PPE que vers les effets de la vie en crèche sur le développement cognitif et affectif des enfants. Il s'agit surtout d'expérimentations menées dans le cadre de pédagogies actives et coopératives (Hardy, Platone, \& Stamback, 1991 ; Pueyo, 1999 ; Meirieu, 2000 ; Stambak, 2000) ou de formations-actions (Pirard, 1997 ; Mauvais, 2000). D'autres recherches ont été centrées sur les aspects spécifiques de l'accueil en crèche par rapport aux pratiques du milieu familial (Donati, Mollo, Norvez, \& Rollet, 1999 ; Zaouche-Gaudron, Ricaud-Droisy, \& Beaumatin, 2002) et sur l'intégration sociale des enfants (Baudelot, Rayna, \& Guibert, 2000 ; Vérillon, Belmont, Vial, \& Portelli, 2000)

Aussi, l'évaluation de la qualité de l'accueil se heurte à l'absence de description des pratiques, contraintes et astreintes que les personnels doivent gérer par rapport à leurs donneurs d'ordre, aux parents et aux enfants. Cette difficulté est permanente dans toutes les relations de service dont l'exigence d'évaluation est récurrente dans une perspective gestionnaire «au nom du réalisme, de l'équité, de la rentabilité voire de la qualité totale » (Dejours, 2001 ; Hubault, \& Bourgeois, 2002). Dans la situation présente, on ne peut espérer mesurer la qualité finale du service rendu aux enfants comme on le ferait d'un produit industriel puisqu'il s'agit d'un « produit immatériel » résultant d'un processus à long terme ( 3 ans) et dont le développement se poursuit bien au-delà du départ de la crèche.

De plus, dès la formulation de la demande, plusieurs niveaux et centres de décision et d'action sur lesquels nous reviendrons $(\$ 4)$ sont apparus comme participant à des degrés divers au fonctionnement des crèches. Dès lors, on peut faire l'hypothèse que la qualité de l'accueil résulte d'un travail collectif dont il faut analyser le processus.

Nous situant dans une perspective ergonomique, il ne s'agit pas de juger des compétences des différents acteurs mais de comprendre les contraintes auxquelles ils sont soumis et leurs possibilités d'y faire face. Pour cela, l'analyse des différentes modalités de régulation du processus de travail mises 
en œuvre par les diverses parties prenantes a permis de préciser un certain nombre de conditions participant à la qualité de service en crèche.

\section{3.- Intérêt scientifique : une approche pluridisciplinaire des régulations du processus de travail en crèche}

L'intérêt scientifique de cette recherche est de proposer une approche des activités de service en crèche qui prenne en compte l'influence des contraintes des situations de travail sur les interactions entre les auxiliaires de puériculture et les enfants. Cette orientation a conduit à développer une analyse pluridisciplinaire des modalités de régulation du processus de travail dans deux perspectives complémentaires, l'une tournée vers les composantes organisationnelles du travail et l'autre orientée vers l'analyse ergonomique de l'activité (Maggi, 1999).

Sans entrer dans les détails des différents aspects de la notion de « régulation » en psychologie du travail (Weill-Fassina, 1971-1972), un bref résumé des divers sens de ce concept selon les disciplines permettra d'expliquer l'opportunité de ce double point de vue sur le travail en crèche.

Dans le sens le plus général, une régulation est «l'action de régler, le fait de maintenir en équilibre, d'assurer le fonctionnement correct d'un système complexe » (Robert, 1994).

Dans une acception classique, l'équilibre recherché est stable et consiste à revenir à l'équilibre de départ. Ainsi, d'un point de vue biologique et physiologique, la régulation est un processus qui vise à assurer la constance ou la stabilité du milieu intérieur en dépit des variations du milieu extérieur. Cette recherche de stabilité est également une caractéristique des régulations dans le domaine cybernétique dont le prototype est le thermostat.

Cependant, d'autres régulations par rapport au milieu impliquent non pas un retour à une norme stable ou stabilisée mais une évolution de l'équilibre pour s'adapter autant que faire se peut à la variabilité des circonstances. Ainsi, des modèles différents et complémentaires ont été élaborés pour décrire les régulations au niveau du processus social de travail, des comportements opératoires face aux exigences des tâches et des conduites psychologiques face aux perturbations du milieu.

D'un point de vue social et organisationnel, la régulation est l'ensemble des règles qui assurent le bon fonctionnement du système. Rappelons les définitions et quelques caractéristiques pertinentes pour cette recherche en nous appuyant sur la théorie de «l'agir organisationnel » (Maggi, 1996, 2003).

- «Les règles du processus d'actions et de décisions sont variables, formelles et informelles, explicites et tacites, conscientes et non conscientes, préalables et intrinsèques à l'action. Les règles sont posées, réélaborées, construites au cours du développement du processus. Ce travail des règles est la régulation (...) du processus.

- Les règles de toute nature sont produites de manière hétéronome ou autonome aux différents niveaux de décision du processus. Elles peuvent impliquer de l'imposition ou de la discrétion (...).

- La régulation du processus d'action concerne la coordination des actions et la coordination des accomplissements des actions.

- L'évaluation du processus d'action concerne la congruence des variabilités de ses composantes, intégrant la congruence par rapport au bien-être des sujets agissants ».

D'un point de vue ergonomique, l'accent est davantage mis sur les modifications du comportement individuel et collectif des opérateurs pour faire face aux exigences des situations. La régulation désigne le processus mis en œuvre par les opérateurs pour construire des compromis entre contraintes antagonistes (Faverge, Olivier, Delahaut, Stephaneck, \& Falmagne, 1970 ; Faverge, 1979-80). 
Qu'il s'agisse, par exemple, de charge de travail (Sperandio, 1972) ou de pression temporelle (De la Garza, Maggi, \& Weill-Fassina, 1999), les régulations réalisées ont pour but de répondre aux obligations de production, de réduire les risques d'erreurs, d'incidents, d'accidents ou de débordements. De même, on a pu montrer, dans différents secteurs, les régulations individuelles et collectives mises en œuvre par les opérateurs pour compenser ou éviter des déficiences physiques ou des risques d'atteinte à la santé tout en maintenant les exigences de la production (Pueyo, \& Gaudart, 2000). Ces régulations se manifestent généralement par des modifications des modes opératoires tout en restant dans les espaces de tolérance du système (Valot, Weill-Fassina, Guyot, \& Amalberti, 1996). Les compromis dépendent des buts et des moyens disponibles ainsi que de la nature de la situation et des responsabilités de chacun. Ils dépendent à la fois des marges de manœuvre dont disposent les opérateurs, de la zone d'initiative et de tolérance qu'ils ont pour assurer un fonctionnement efficace du système et aussi de leurs capacités à s'en saisir (Weill-Fassina, \& Valot, 1998). Ce sont souvent des ajustements, des adaptations, des détournements, des transformations de procédures (Caroly, 2002). «Il ne s'agit pas d'une rupture de la règle et de son application, mais (...) d'un moyen de résoudre des contradictions, des conflits obligeant (l'opérateur) à trouver des compromis » pour que le système continue à fonctionner dans les meilleures conditions possibles pour les différentes parties concernées (Caroly, \& Clot, 2004).

On peut les réinterpréter au niveau organisationnel comme des stratégies sous-tendues par des réélaborations plus ou moins implicites de règles. A la limite, l'échec réel ou ressenti de ces régulations individuelles ou collectives peut être marqué par des réaffectations au sein d'une même entreprise, voire par des exclusions. Cette «régulation organisationnelle », selon l'expression employée par Gonon (2003), semble se situer à un autre niveau que la régulation du processus de travail définie plus haut. Dans l'étude citée, elle ne porte pas sur «l'ensemble des règles qui assurent le bon fonctionnement du système » mais sur le parcours professionnel d'infirmières dans le cadre d'un système plus large que celui considéré ici, puisqu'un centre hospitalier offre en principe des possibilités de changements de poste voire de métiers. Elle concerne donc la gestion de l'emploi et des ressources humaines de l'entreprise pour répondre à ses besoins d'efficacité et/ou aux demandes du personnel qui ont du mal à faire face aux exigences de leur travail. Ce type de régulation a effectivement émergé dans les crèches sous forme de turn-over des auxiliaires (cf. §9). Nous l'avons considéré comme un critère des difficultés rencontrées sans toutefois l'analyser dans la mesure où nous étions centrés sur les articulations des régulations des services rendus aux enfants dans le cadre d'un processus de travail donné.

Dans une perspective psychologique, les régulations sont des réactions du sujet à des perturbations et visent à les compenser en reconstruisant l'équilibre du système d'activités. Selon la théorie piagétienne de l'équilibration (Piaget, 1975), les exigences des tâches peuvent être ressenties comme des sources de déséquilibre ou de perturbations qui font obstacle à l'assimilation des situations et à l'atteinte du but. Elles impliquent de la part du sujet une reconstruction ou une restructuration de ses connaissances, savoirs, savoir-faire dans le cadre de ses interactions avec le milieu pour agir sur lui, l'utiliser ou le transformer. Cependant, si toute régulation est une réaction à une perturbation, toute perturbation n'entraîne pas une régulation et toute régulation n'aboutit pas à une compensation complète de la situation. Inhelder (1987) distingue à ce propos :

- «les compensations neutres » : l'opérateur ignore la perturbation, la refuse, n'intervient pas, ne tente aucune régulation ;

- «les compensations partielles » : l'opérateur adopte une solution de compromis entre sous-systèmes contradictoires sans que soit pris en compte (volontairement ou non) l'ensemble des paramètres de la situation;

- « les compensations totales » qui prennent en compte l'ensemble des paramètres et des possibles de la situation. 
L'articulation de ces trois points de vue sur les modalités de régulation du processus de travail a paru particulièrement féconde pour interpréter les activités de service en crèche dans leurs relations avec le processus organisationnel et poser des hypothèses sur le fonctionnement d'ensemble de ce système et notamment sur les coordinations des différents centres de décision impliqués.

\section{4.- Les centres de décisions et d'actions impliqués dans le fonctionnement des crèches}

Les buts, les moyens et les tâches en crèches sont définis à plusieurs niveaux de responsabilité par plusieurs centres dans un enchaînement de décisions et actions supposées coordonner le processus de travail de sa conception à sa réalisation. Nous avons analysé ces différents centres de décision à la lumière des trois modalités de régulation dominantes que les acteurs du système mettent en œuvre pour optimiser son fonctionnement (Figure 1)

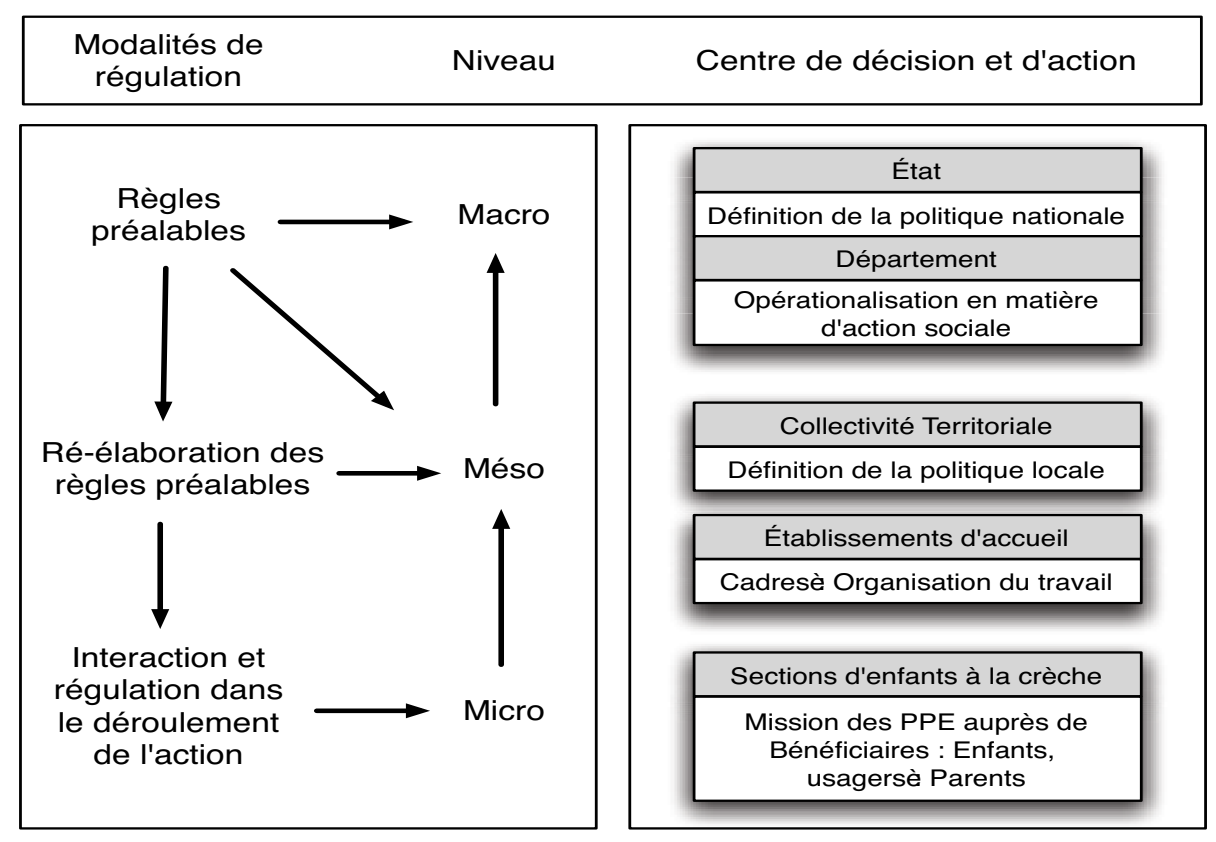

Figure 1.- Modalités de régulation selon les principaux centres de décisions et d'actions intervenant dans le processus de travail en crèche

- Au niveau institutionnel « macro » le plus global, l'Etat, par l'intermédiaire de différents ministères, définit la politique nationale à l'égard de la Petite Enfance ; des lois, décrets et circulaires définissent les principales règles préalables et les normes de fonctionnement du secteur : but, conditions d'exécution et moyens disponibles. Le département est chargé de l'opérationnalisation de ces règles en matière d'action sociale. Il s'agit surtout des modes de garde de la petite enfance, des règles sanitaires et de protection de la mère et de l'enfant, des conditions d'attribution de la délivrance des agréments pour les formes d'accueil collectif des missions des PPE, de la formation, des droits et devoirs des personnels.

- A un niveau institutionnel intermédiaire, "meso », la collectivité locale (Mairie) a pour responsabilité la définition de la politique locale des structures municipales d'accueil des jeunes enfants qu'elle propose à la population, suivant le «Contrat Enfance » de la Caisse d'Allocations Fami- 
liales. Elle décide de l'ouverture des crèches, assure la gestion du personnel en appliquant la législation du travail en vigueur et décide entre autres des effectifs et des heures d'ouverture et de fermeture des crèches.

- A un niveau "meso » plus directement managérial, la Direction des crèches décide de l'organisation du travail dans l'établissement: horaires d'ouverture en accord avec la Mairie, projet pédagogique, règlement intérieur en accord avec le personnel et les parents. Les Directrices animent les réunions pédagogiques, veillent au bon fonctionnement de la crèche et gèrent les éventuels conflits avec la Mairie, entre les membres de l'équipe ou avec les parents.

- Au niveau «micro », en section, les activités de service sont constituées par les interactions entre auxiliaires de puériculture, parents et enfants. Leurs missions principales, auprès des parents et des enfants (Figure 2), laissent prévoir de nombreux échanges avec ces différents interlocuteurs et des régulations de leurs comportements pour faire face à la multiplicité de leurs tâches.

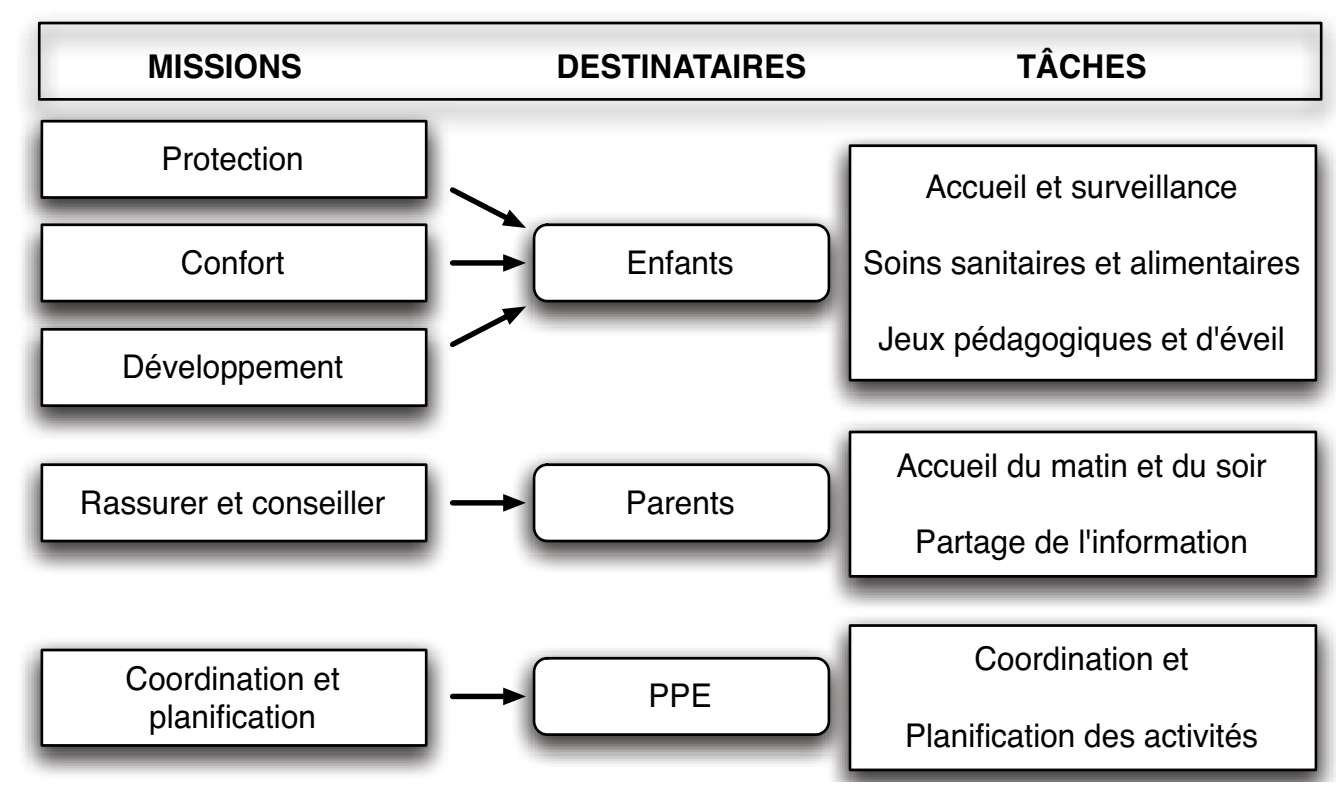

Figure 2.- Missions des Auxiliaires de puériculture

Les parents et les enfants constituent deux autres centres de décisions et d'action avec un statut particulier en ce sens qu'ils n'établissent pas des règles de fonctionnement mais manifestent des attentes et des besoins dont la satisfaction constitue le but de toute l'organisation précédente.

- Les parents en sont les «usagers» dans la mesure où ils utilisent le service offert et ne «l'achètent pas »; la contribution financière demandée est modulée selon leurs revenus. Leurs attentes varient avec le milieu social des familles (Cristofari, \& Miranda,1988) : les plus favorisées attendent une approche pédagogique et sanitaire pour leurs enfants, tandis que les plus modestes attendent un suivi plutôt sanitaire. Elles attendent aussi que, dans le cadre du projet pédagogique institutionnel, les PPE apprennent à l'enfant à devenir propre et à être plus autonome (Marcos, Orvig-Salazar, Bernicot, Guidette, Hudelot, \& Preneron, 2000). Les familles peuvent déléguer à la crèche leurs fonctions et responsabilités vis-à-vis de l'enfant, totalement, de manière partagée ou conflictuelle. Les demandes des familles d'être accompagnées dans leurs relations avec leurs enfants, d'être rassurées et de pouvoir échanger sur leurs difficultés, constituent de nouvelles attentes qui s'expriment surtout auprès des crèches collectives (Bouve, 2001). 
Les parents peuvent cependant agir sur les processus de décisions par leurs exigences ou leurs appuis dans les démarches auprès des collectivités locales comme le prouvent certains courriers ou pétitions.

- Les enfants sont les bénéficiaires du service : ils sont dans un processus visant leur développement et leur autonomie. Les actions des auxiliaires ont pour but d'agir sur leurs conditions de vie, leur personnalité, de les protéger, de les éduquer et de les socialiser, dans une relation interactive avec leurs besoins et leurs possibilités (Dubet, 2002). Or, ceux-ci évoluent avec l'âge des enfants d'une année sur l'autre et même au cours de l'année, au fur et à mesure de leur développement physique, intellectuel et psychologique. Les activités des auxiliaires de puériculture devront répondre à leurs attentes, besoins et capacités à la fois de manière individualisée et collective dans le groupe qu'ils constituent dans chaque section.

Ce panorama des différents centres de décision et d'action permet de caractériser plusieurs aspects du cadre institutionnel et managérial dans lequel se déroulent les activités des PPE. Même si le but est commun, les règles et les exigences ont des sources qui ne sont pas toutes en interactions.

- Entre instances législatives et administratives, les relations sont plutôt à dominante descendante ; les retours d'informations sont probablement rares et se font en « régulation froide », hors des activités de service (de Terssac, 1992).

- Entre collectivité locale et structure d'accueil, les possibilités d'échanges risquent d'être sporadiques et conflictuelles. C'est en partie en raison de tels désaccords que l'intervention ergonomique a eu lieu.

- Il en est de même entre la Mairie et les usagers qui se trouvent être aussi des citoyens.

- Au contraire, dans les structures d'accueil, les Cadres de Direction, les Auxiliaires, les Parents, les Enfants ont des échanges et des communications directs permettant éventuellement des régulations « à chaud » des situations difficiles dans le déroulement-même de l'activité.

Cette description schématique des fonctions et des relations des centres de décision participant à l'organisation, à la gestion et à la réalisation des activités de services en crèche permet de poser les hypothèses suivantes quant aux diverses modalités de régulation du processus de travail :

1. Malgré leur but commun, il pourrait y avoir des problèmes de coordination des décisions et actions des instances institutionnelles qui seraient à la source de dysfonctionnements notamment en ce qui concerne les absences.

2. Dans ce cas, les règles préalables à l'action feraient l'objet de réélaborations au niveau de l'encadrement de la crèche pour assurer un fonctionnement optima par rapport aux moyens disponibles et aux besoins des parents et enfants. Mais les compromis mis en place pour réguler les incompatibilités entre règles, les décalages entre moyens et buts, les astreintes du système ne compenseraient que partiellement le dysfonctionnement.

3. Dans ce contexte, du point de vue ergonomique, les régulations des activités en section se caractériseraient par des modifications des comportements des auxiliaires de puériculture vis-à-vis des enfants, modifications que nous caractériserons en fonction des astreintes de la situation.

\section{5.- Une recherche participative}

En raison même des décalages constatés dès l'analyse de la demande, les modalités d'intervention ont été structurées de manière largement participative pour favoriser les rencontres et les discussions entre les représentants de la collectivité locale, les personnels des crèches et les usagers, en les sollicitant pour le recueil des informations, le contrôle de leur interprétation et la mise en place de leur exploitation. Trois groupes ont été constitués. Ce qui a permis de faire émerger un collectif fondé sur la confiance entre les participants et les intervenants. 
- Un groupe de pilotage, composé de l'élu municipal responsable de la petite enfance, de la coordonnatrice de la petite enfance, des représentants de la Direction des Ressources Humaines et des autres services concernés, des membres volontaires du groupe de travail et des ergonomes, a assuré le guidage global de l'intervention et la validation des résultats en vue de leur utilisation.

- Un groupe de suivi, composé des cadres de direction volontaires appartenant aux diverses structures d'accueil de la municipalité, a participé à l'étude par un travail collectif effectué en réunions plénières et un travail individuel réalisé dans leur structure respective, dans le but de relever, systématiser et comparer des informations concernant le fonctionnement de leur établissement respectif.

- Un groupe de travail, dans une crèche, constitué de la Directrice, l'Adjointe, la Psychologue, le Médecin et, pour chaque section, une Auxiliaire de puériculture et une Educatrice, avait pour mission d'interagir en continu avec l'ergonome, de contribuer à la compréhension de la situation, de valider les résultats, de proposer et d'expérimenter des solutions possibles.

Une activité de communication, de négociation, de restitution et de confrontation en continu des résultats a permis de tenir tous les partenaires de la recherche y compris les parents, au courant de son déroulement (Gonzales, \& Teiger, 2001) afin de mettre en commun leurs connaissances sur le fonctionnement des crèches et les sources de dysfonctionnement sur lesquels il était possible d'agir.

\section{6.- Des méthodes d'analyse complémentaires}

La méthodologie d'analyse a mis en œuvre plusieurs techniques complémentaires pour caractériser les modalités de régulation des différents centres de décision.

- Au niveau « Macro », une étude documentaire et des entretiens individuels ont permis d'accéder aux règles préalables essentielles.

- Au niveau «Méso », une formation-action constituée de 10 réunions soit 600 minutes d'enregistrement entièrement transcrites (Gonzalez, \& Teiger, 2001), des réunions pédagogiques et des entretiens collectifs, également enregistrés et transcrits, ont permis aux participants de prendre conscience du fonctionnement réel des crèches de la commune. Cette approche a permis de mettre en évidence les règles préalables de la collectivité, les difficultés rencontrées et les réélaborations de ces règles pour optimiser le fonctionnement des crèches.

- Au niveau «Micro », des observations systématiques des interactions des auxiliaires de puéricultures avec les parents et les enfants au cours de l'accueil, du goûter, du repas et des jeux pédagogiques, ont été réalisées dans les sections de bébés, moyens et grands. Les enregistrements vidéo ont permis de mettre en évidence les spécificités de leurs activités et les modalités de régulations de leur comportement en cas de surcharge de travail.

\section{7.- Des règles institutionnelles préalables contradictoires : Cadre temporel, taux d'encadrement et tâches}

La confrontation des règles issues des différentes instances institutionnelles a mis en évidence des contradictions entre règles préalables concernant le cadre temporel, le taux légal d'encadrement et les tâches à réaliser.

- Le cadre temporel est défini par le «temps de l'horloge ». "C'est un temps extérieur au sujet, extérieur à l'action qui peut servir tantôt de mesure, tantôt de repère, tantôt de contrainte puisque c'est en même temps le temps organisationnel » (De Keyser, 1990 ; Grossin, 1996). Il s'agit ici du temps de travail, des horaires d'ouverture et de fermeture de la crèche, des horaires du personnel et de la division de la journée selon les tâches à accomplir. 
- Le taux d'encadrement est défini comme le nombre d'auxiliaires de puériculture par rapport au nombre d'enfants. Selon la règle préalable, ce taux est de 1 PPE pour 5 enfants qui ne marchent pas en section de bébés et de 1 PPE pour 8 enfants qui marchent en section de moyens et de grands. Ce qui correspond aux « ressources humaines » affectées.

- Les tâches impliquées par les différentes missions (Figure 2) sont diversifiées et planifiées pour la journée : accueil, goûter, repos, déjeuner, jeux nécessitent plus ou moins de monde.

L'analyse de la coordination entre ces trois catégories de règles préalables fixées au niveau national et municipal fait apparaître en situation nominale des incompatibilités quant à la possibilité d'assurer le taux d'encadrement légal dans les sections des crèches.

Pendant la journée,

- Au niveau national, la durée quotidienne de travail est fixée par l'Etat à 35 heures / semaine soit 7 heures par jour pour une semaine de 5 jours.

- Au niveau municipal, l'amplitude d'ouverture des crèches est de 12 heures

- Au niveau de la crèche, étant donné le taux d'encadrement fixé au niveau national et le personnel affecté en crèches, soit 5 PPE par section de 20 enfants, pour couvrir l'amplitude horaire de la crèche, en respectant la durée de travail légale, les arrivées et les départs du personnel doivent se faire en horaire décalé. Dans une section, la première PPE vient à $7 \mathrm{~h} 30$ du matin la seconde à $8 \mathrm{~h} 30$ et les 3 suivantes toutes les demi-heures jusqu'à 10h30. Les départs ont lieu selon le même principe à partir de $15 \mathrm{~h} 30$.

- Cependant, au niveau national, un taux minimum de présence de deux PPE dans une section est requis. Dans le cas des horaires décalés, étant donnés les horaires d'arrivée des parents pour amener ou chercher leur enfant, ces deux règles préalables concernant le taux d'encadrement ne peuvent pas toujours être respectées. Par exemple, on a pu observer entre $7 \mathrm{~h} 30$ et $9 \mathrm{~h} 30$ en section bébés 1 PPE pour 8 enfants, puis 2 PPE pour 18 enfants.

En ce qui concerne les congés,

- Au niveau national, les congés annuels sont fixés à 33 jours par an, soit $11 \%$ d'absences par an par rapport au nombre de jours ouvrés de l'établissement. Ces congés sont non remplacés.

- Au niveau de la crèche, il est convenu que les congés soient pris à tour de rôle pendant toute l'année, mais ils diminuent aussi le taux d'encadrement.

- Au niveau de la crèche, les réunions pédagogiques sont prévues en heures supplémentaires non rémunérées. Ces heures sont à rattraper en jours de congés. D'où de nouvelles absences jouant sur le taux d'encadrement. Peuvent s'ajouter dans les mêmes conditions des demandes d'heures supplémentaires à rattraper ultérieurement qui ne font que repousser le problème.

Ces incompatibilités engendrent donc des absences dont nous allons montrer l'origine structurelle et des difficultés à assurer au quotidien le taux d'encadrement des enfants, ce qui pose des problèmes tant dans la gestion de la structure d'accueil que dans celle des sections.

\section{8.- Les absences : un dysfonctionnement structurel du processus de travail en crèche}

L'analyse statistique des absences dans une des crèches collectives municipales pendant cinq années scolaires a d'abord permis de dénombrer entre 497 jours et 550 jours d'absence pour 15 personnes. Environ 70\% d'entre elles étaient dues aux congés annuels et à d'autres congés légaux (formation, congés de maternité), le reste étant lié à des congés maladies ou à des congés spécifiques accordés par la Mairie (Tableau 1). 2/3 des absences étaient donc prévisibles, ce qui, en principe, aurait pu permettre de les anticiper et de s'organiser. 


\begin{tabular}{|r|r|r|r|r|r|}
\hline & \multicolumn{7}{|c|}{ Années } \\
\hline \multicolumn{1}{|c|}{ Type d'absence pour congés } & \multicolumn{1}{|c|}{$\mathbf{9 6 - 9 7}$} & $\mathbf{9 7 - 9 8}$ & $\mathbf{9 8 - 9 9}$ & $\mathbf{9 9 - 0 0}$ & $\mathbf{0 0 - 0 1}$ \\
\hline Prévisibles Annuels & & & & & \\
\hline Formation & 62,3 & 49,7 & 60,5 & 61.5 & 62.4 \\
\hline Maternité & 5,1 & 2,2 & 0,6 & 0.5 & 0.8 \\
\hline Total & 0,0 & 18,4 & 7,9 & 10,0 & 10,0 \\
\hline Non-Prévisibles & 67,5 & 70,4 & 69,1 & 72,0 & 73.2 \\
\hline \multicolumn{1}{|r|}{ Congés de la Mairie } & 18,7 & 15,7 & 19,6 & 17.6 & 16.7 \\
\hline Total & 13,8 & 14,0 & 11,3 & 10.4 & 10.1 \\
\hline
\end{tabular}

Tableau 1.-Pourcentage des différentes causes d'absences dans une crèche collective durant cinq années

Cette première analyse a souligné que la grande majorité des absences du personnel avaient eu des raisons structurelles plus que conjoncturelles et individuelles. Faute de remplacements possibles, cela laissait prévoir de nombreux cas de fonctionnement en section en situation dégradée, ce qu'a confirmé une analyse d'ensemble de la situation des crèches de la commune. Les Cadres de Direction de deux crèches et deux mini-crèches de la collectivité locale concernée ont relevé les présences et absences du personnel et des enfants dans les différentes sections pendant 20 jours ouvrables d'un même mois. Soit, au total, 160 jours de relevés.

Sur la base de ce bilan, quatre situations-types d'encadrement par journée, ont été définies, en tenant compte des horaires décalés.

- Situation standard: le nombre d'adultes et d'enfants est égal au nombre prévu ;

- Situation favorable : le nombre d'adultes est égal au nombre prévu et le nombre d'enfants se situe entre la moitié et les $2 / 3$ des inscrits ;

- Situation dégradée : le nombre d'adultes se situe entre la moitié et les $2 / 3$ prévus et le nombre d'enfants est égal au nombre d'inscrits ;

- Situation intermédiaiere (plutôt défavorable ou plutôt favorable) : le nombre d'adultes se situe entre 70 et $90 \%$ et le nombre d'enfants est égal au nombre inscrits; ou le nombre d'enfants se situent entre 70 et $90 \%$ des inscrits et le nombre d'adultes est égal au nombre prévu.

La répartition des situations journalières pour l'ensemble des trois établissements montre que le taux d'encadrement réel des enfants diffère très souvent du taux nominal (Tableau 2).

\begin{tabular}{|c|c|c|c|c|c|c|c|c|c|}
\hline Situation & \multicolumn{3}{|c|}{ Accueil 1} & \multicolumn{3}{|c|}{ Accueil 2} & \multicolumn{2}{|c|}{ Accueil 3} & Total \\
\hline & Bébés & Moyens & Grands & Bébés & Moyens & Grands & Mini 1 & \begin{tabular}{|l|} 
Mini 2 \\
\end{tabular} & \\
\hline Standard & 4 & 5 & 3 & 2 & 1 & 0 & 0 & 0 & $\begin{array}{r}15 / 160 \\
94 \%\end{array}$ \\
\hline Favorable & 3 & 0 & 0 & 1 & 1 & 3 & 0 & 0 & $\begin{array}{l}8 / 160 \\
50 \%\end{array}$ \\
\hline Dégradée & 4 & 8 & 3 & 9 & 3 & 4 & 5 & 2 & $\begin{array}{l}38 / 160 \\
237 \%\end{array}$ \\
\hline Intermédiaire & 9 & 7 & 14 & 8 & 15 & 13 & 15 & 18 & $\begin{array}{r}99 / 160 \\
61,1 \%\end{array}$ \\
\hline
\end{tabular}

Tableau 2.- Fréquence des situations-types dans les sections de trois structures d'accueil La situation «standard » s'est produite dans moins de $10 \%$ des cas relevés. Le pourcentage de situations favorables qui permettraient éventuellement de « compenser» les aléas survenus dans d'autres sections est faible (5\% des cas). Les mini-crèches sont toujours en situation dégradée ou intermé- 
diaire. En revanche, une situation très perturbée survient une fois par semaine dans l'une ou l'autre des sections et mini-crèches (23,7 \% de cas), avec une variabilité importante entre établissements.

L'étude montre donc la difficulté à assurer le taux d'encadrement fixé par les règles préalables et pose deux questions sur le processus de décision et d'action en crèche :

- Comment les Cadres de Direction régulent-elles les dysfonctionnements liés aux absences pour améliorer autant que faire se peut la qualité du service rendu ?

- Quelles sont les répercussions des situations dégradées par les absences sur le comportement des auxiliaires en section auprès des jeunes enfants ?

\section{9.- Stratégies de régulation managériales : Réélaborations des règles préalables}

Les situations dégradées entraînent des perturbations diverses selon les catégories d'acteurs dans les différents centres de décision qui participent au fonctionnement des crèches.

- L'analyse des réunions et des entretiens montre que les Cadres de Direction ressentent les absences comme un obstacle majeur pour accomplir leurs tâches quotidiennes de surveillance, de prévention, de suivi des enfants et du personnel et de relations avec les parents en raison des remplacements et des réorganisations des équipes en section que ces absences nécessitent dans l'urgence.

- Les Auxiliaires de puériculture soulignent que le travail en sous-effectif, réparti entre les membres présents, entraîne des difficultés de gestion des activités en section pour assurer la qualité, la sécurité et le bien-être des enfants (Cf. §10). A plus long terme, ce mécontentement provoque un turn - over relativement important.

- Les Enfants sont également pénalisés par les absences. Les modifications de leurs habitudes ne permettent pas l'acquisition ou le maintien des repères nécessaires à leur bien-être. De nombreux incidents, enregistrés sur le cahier de transmission d'informations utilisé entre auxiliaires, sont considérés comme une conséquence des absences et des difficultés qu'a le personnel d'assurer une surveillance continue.

- Enfin, les Parents considèrent les conditions d'accueil insuffisantes et réclament en tant qu'usagers à la Mairie une meilleure organisation, comme en témoignent plusieurs courriers.

Pour réguler ces perturbations ressenties comme autant d'astreintes surtout aux moments cruciaux de la journée (accueil, repas), les Cadres de Direction mettent en œuvre une diversité de stratégies dont elles ont fait la liste lors des réunions de groupe.

- Certaines exploitent les marges de manœuvre prévues par les règles préalables. Il s'agit par exemple de la planification à moyen terme des congés prévisibles ou, à plus court terme, de l'appel à l'équipe des auxiliaires volantes de la municipalité.

- D'autres solutions, tout en se situant dans un cadre légal, impliquent un détournement de l'objectif de la règle préalable. Par exemple, faire venir une animatrice du service enfance ou un «emploi-jeune » répond à des règles préalables légales au niveau de l'Etat et de la Collectivité locale mais il s'agit d'un détournement de ces règles parce que ni l'emploi-jeune ni l'animatrice ne sont habilitées à intervenir directement en section (Décret n 2000-762, rt. R. 180-1).

- D'autres solutions sont des réélaborations de règles managériales concernant le fonctionnement de l'établissement. Modifier les heures d'ouverture et fermeture sans l'accord de la Mairie marque une prise d'autonomie par rapport à la collectivité locale. Créer une PPE volante interne à la crèche, regrouper dans une même salle l'accueil des trois sections le matin et le soir, constituent des décisions prises à leur discrétion. 
- Enfin, d'autres réélaborations de règles managériales concernent la gestion du personnel : par exemple, proposer à une PPE d'aller dans une autre section ou de récupérer les heures supplémentaires à un autre moment que celui prévu.

L'analyse de contenu des discussions en réunions pédagogiques a montré que ces régulations des situations dégradées aboutissent à des compensations partielles qui ne satisfont personne car les réorganisations mises en place engendrent d'autres types des problèmes.

- Faire venir une personne extérieure à l'établissement est une aide limitée parce que celle-ci ne connaît ni les enfants ni les tâches des PPE, d'où une limitation du type d'aide et une surcharge des auxiliaires présentes par les explications à donner.

- Proposer une auxiliaire volante interne ou le changement de section à une PPE transfère le problème d'une section à l'autre en déséquilibrant l'effectif de l'autre section, en provoquant l'abandon des enfants référents et produit dans la section aidée une surcharge en augmentant les renseignements à chercher et à donner sur des enfants qu'elle ne connaît pas.

- Par rapport aux enfants, ces deux types de solution risquent de provoquer des pertes de repères, et des nécessités de réadaptation qu'il faut gérer.

- Réduire les heures d'ouverture et de fermeture de l'établissement, provoque le mécontentement des PPE et des parents en raison des interférences avec leur vie de famille ou de travail et celui des cadres politiques et administratifs de la mairie auxquels des plaintes sont adressées.

\section{0.- Régulations des activités en section : Modifications des comportements en fonction des astreintes}

Les difficultés dues à un taux d'encadrement inférieur à la norme entraînent selon les PPE un surcroît de travail en section. En nous appuyant sur les recherches sur les effets d'une surcharge de travail (Sperandio, 1972), nous avons fait l'hypothèse que cette perturbation pouvait engendrer des modifications du comportement des auxiliaires en lien avec celui des enfants au cours des différentes activités.

Pour vérifier cette hypothèse, nous avons comparé 12 séquences en «situation standard » et 12 en « situation dégradée » à raison d'une séquence de 20 minutes par activité et par section d'observations selon le plan décrit au tableau 3.

D'un point de vue théorique, l'analyse des comportements s'inspire des études sur les relations de service menées dans une perspective interactionniste dans les secteurs administratifs et marchands (Weill-Fassina, \& Caroly, 2003). Elle s'en distingue sur deux points essentiels en raison des spécificités des relations psycho-socio-pédagogiques.

- L'analyse de contenu de ces enregistrements a été centrée sur les interventions des auxiliaires en considérant les agissements des enfants dans leur contexte de groupe. Ce sont des communications verbales, des actions, des gestes, des regards qui s'étendent au groupe et ne sont pas circonscrits dans un tête-à-tête.

- L'analyse a permis de spécifier les dimensions de ces interventions par analogie avec les trois dimensions traditionnellement retenues pour caractériser les communications dans les relations de service (Goffman, 1968 ; Falzon 2000). Rappelons que ces trois dimensions sont les dimensions technique (ou transactionnelle) visant à résoudre le problème, contractuelle (ou organisationnelle) correspondant aux règles de l'entreprise et civiles (ou relationnelles) relevant de la politesse et du contact avec le client. 
La transposition de ces dimensions à la situation en section a conduit à distinguer dans les interventions des auxiliaires trois dimensions principales qui peuvent prendre des «tonalités » différentes selon les buts poursuivis en situation.

\begin{tabular}{|c|c|c|c|c|}
\hline $\begin{array}{l}\text { Activités } \\
\text { Section }\end{array}$ & Accueil & Jeux d'éveil & Déjeuner & Goûter \\
\hline $\begin{array}{l}\text { Bébés } \\
\text { Groupe }\end{array}$ & $\begin{array}{c}1 \text { auxiliaire, } 5 \text { enfants, } \\
3 \text { parents }\end{array}$ & $\begin{array}{l}\text { 1 éducatrice, } \\
5 \text { enfants, }\end{array}$ & 1 auxiliaire, 5 enfants & 1 auxiliaire, 5 enfants \\
\hline $\begin{array}{c}\text { d'enfants de } 3 \text { à } \\
12 \text { mois }\end{array}$ & $\begin{array}{c}2 \text { auxiliaires, } 18 \text { enfants, } \\
3 \text { parents }\end{array}$ & $\begin{array}{l}1 \text { éducatrice, } \\
12 \text { enfants }\end{array}$ & $\begin{array}{l}1 \text { auxiliaire, } \\
10 \text { enfants }\end{array}$ & $\begin{array}{l}2 \text { auxiliaires, } \\
18 \text { enfants }\end{array}$ \\
\hline $\begin{array}{l}\text { Moyens } \\
\text { Groupe }\end{array}$ & $\begin{array}{c}1 \text { auxiliaire, } 8 \text { enfants, } \\
3 \text { parents }\end{array}$ & $\begin{array}{l}\text { 1 éducatrice, } \\
8 \text { enfants }\end{array}$ & 1 auxiliaire, 8 enfants & 1 auxiliaire, 8 enfants \\
\hline $\begin{array}{l}\text { d'enfants de } 12 \text { à } \\
24 \text { mois }\end{array}$ & $\begin{array}{c}2 \text { auxiliaires, } 19 \text { enfants, } \\
3 \text { parents }\end{array}$ & $\begin{array}{l}1 \text { éducatrice, } \\
12 \text { enfants }\end{array}$ & $\begin{array}{l}1 \text { auxiliaire, } \\
10 \text { enfants }\end{array}$ & $\begin{array}{l}1 \text { auxiliaire, } \\
10 \text { enfants }\end{array}$ \\
\hline $\begin{array}{l}\text { Grands } \\
\text { Groupe }\end{array}$ & $\begin{array}{c}1 \text { auxiliaire, } 8 \text { enfants, } \\
3 \text { parents }\end{array}$ & $\begin{array}{l}1 \text { éducatrice, } \\
8 \text { enfants }\end{array}$ & 1 auxiliaire, 8 enfants & 1 auxiliaire, 8 enfants \\
\hline $\begin{array}{l}\text { d'enfants de } 24 \text { à } \\
36 \text { mois }\end{array}$ & $\begin{array}{c}1 \text { auxiliaire, } 18 \\
\text { enfants, } 3 \text { parents }\end{array}$ & $\begin{array}{l}1 \text { éducatrice, } \\
12 \text { enfants }\end{array}$ & $\begin{array}{c}1 \text { auxiliaire, } \\
10 \text { enfants }\end{array}$ & $\begin{array}{c}1 \text { auxiliaire, } \\
10 \text { enfants }\end{array}$ \\
\hline
\end{tabular}

Tableau 3.- Plan de comparaisons des situations

- La dimension pédagogique réfère aux interventions dont le but est d'apprendre à un enfant ou au groupe une technique de jeux, les principes éducatifs de la crèche ou à agir sur un objet, à l'aider, ... Cet aspect, qui correspond à la dimension technique précédemment citée, prend plus ou moins d'importance selon les sections.

- La dimension relationnelle réfère aux rapports sociaux individualisés entre l'auxiliaire et un enfant donné. La transposition de ces dimensions à la situation en section nous a conduit à distinguer, dans les interventions des auxiliaires, trois dimensions principales qui peuvent prendre des «tonalités » différentes selon les buts poursuivis en situation et se déclinent en plusieurs tonalités selon les circonstances.

- Relationnelle - encouragement, elle vise à inciter l'enfant à agir ou à le féliciter pour l'habileté dont il fait preuve à tel ou tel moment.

- Relationnelle - protectrice, elle vise à protéger un enfant en réaction aux risques qu'il encourt du fait de son comportement ou du comportement d'autres enfants.

- Relationnelle - corrective : elle vise à faire acquérir à un enfant des règles de vie en collectivité par des remarques critiques en réaction à son comportement social inadéquat par rapport au groupe. 


\begin{tabular}{|c|c|c|}
\hline Dimension de l'intervention & Tonalités & Exemples \\
\hline Pédagogique & & $\begin{array}{l}\text { - Montrer comment prendre sa fourchette, sa cuillère, son } \\
\text { verre, se servir à table, } \\
\text { - Montrer ou expliquer comment jouer avec un ballon, } \\
\text { une voiture, un objet, un livre, un puzzle..., } \\
\text { - Laisser choisir une activité, } \\
\text { - Enseigner la politesse, } \\
\text { - Expliquer les actions à suivre... }\end{array}$ \\
\hline \multirow{3}{*}{ Relationnelle } & Protectrice & $\begin{array}{l}\text { - Proposer de l'aide à l'enfant en difficulté, } \\
\text { - L'aider à monter pour éviter qu'il ne tombe, } \\
\text { - Le descendre d'un endroit d'où il risque de tomber, } \\
\text { - Demander à un enfant d'arrêter une action parce qu'il en } \\
\text { met un autre en danger. }\end{array}$ \\
\hline & Encouragement & $\begin{array}{l}\text { - Donner ou aider à manger, } \\
\text { - Faire des compliments à voix haute, } \\
\text { - Embrasser, câliner, consoler, chatouiller, cajoler, rassu- } \\
\text { rer. }\end{array}$ \\
\hline & Corrective & $\begin{array}{l}\text { - Demander d'arrêter de prendre les jouets des autres, } \\
\text { - Demander d'arrêter un comportement qui dérange, } \\
\text { - Ordonner d'arrêter de se disputer entre eux, } \\
\text { - Ordonner d'attendre son tour. }\end{array}$ \\
\hline \multirow{2}{*}{ Organisationnelle } & Proactive & $\begin{array}{l}\text { - Aménager la section, les lits, les tables, les jouets, la } \\
\text { salle de bains, le dortoir, } \\
\text { - Organiser le lieu ou les jouets pour les jeux prévus, } \\
\text { - Préparer le repas, le yaourt, les fruits..., } \\
\text { - Placer les enfants à table selon leur santé,,leur compor- } \\
\text { tement,,leur autonomie... }\end{array}$ \\
\hline & Réactive & $\begin{array}{l}\text { - Demander d'arrêter de pleurer, de crier, de déranger les } \\
\text { autres, } \\
\text { - Éloigner un enfant qui empêche les autres de réaliser } \\
\text { une activité, } \\
\text { - Enlever un jouet, un objet à un enfant pour le redonner à } \\
\text { un autre auquel il l'avait pris. }\end{array}$ \\
\hline
\end{tabular}

Tableau 4.-Catégorisation de quelques interventions des auxiliaires auprès des enfants selon leurs dimensions et leurs tonalités

- La dimension organisationnelle réfère à l'organisation du groupe d'enfants et à l'aménagement des conditions sociales ou matérielles des différentes activités. Il s'agit des « règles du jeu » collectives que les auxiliaires mettent en place dans leur section et non des règles ou missions qui leur sont confiées et qui deviennent implicites dans cette analyse. Cette dimension diffère donc de ce que l'on entend habituellement par «dimension contractuelle ou organisationnelle ». Les tonalités portent sur les possibilités de planification qu'ont les auxiliaires en situation.

- Organisationnelle - proactive : cette tonalité recouvre des actions qui visent à anticiper des situations préparer une activité et/ou prévoir des comportements potentiellement perturbateurs pour son bon déroulement.

- Organisationnelle - réactive : cette tonalité recouvre des interventions au coup par coup qui, suite à l'apparition de comportements perturbateurs, tendent à réaménager la situation dans le groupe pour que les activités en cours puissent continuer.

Le tableau 4 donne quelques exemples de catégorisation des interventions en fonction des dimensions et des tonalités concernées. 
Plusieurs dimensions peuvent être mises en œuvre simultanément. Par exemple, une intervention relationnelle corrective pour un enfant peut en même temps être une intervention relationnelle protectrice pour un autre.

Ces dimensions et leurs tonalités, considérées comme symptôme de la qualité du rapport avec les enfants compte tenu de l'atteinte du but de l'activité en cours, peuvent être regroupées en deux grandes catégories :

- La dimension pédagogique, les tonalités relationnelle - encouragement et organisationnelle proactive indiqueraient une "régulation maîtrisée » de la situation avec du temps pour expliquer, câliner, planifier , anticiper...

- Les tonalités relationnelle - protection, relationnelle - corrective ou organisationnelle - réactive des interventions (en grisé sur le tableau) indiqueraient une « régulation opportuniste » au coup par coup, d'événements plus ou moins critiques survenant dans le groupe d'enfants au cours des différentes activités.

Les résultats (Figure 3) montrent qu'en situation nominale, le pourcentage des «régulations maîtrisées» varie d'une activité et d'une section à l'autre. Est implicite, en complément sur la figure, la présence de «régulations opportunistes» lors d'interventions dues à des besoins plus ou moins intempestifs ou à des initiatives plus ou moins heureuses des enfants.

Les situations dégradées, conformément aux hypothèses, sont marquées par une diminution des pourcentages de « régulations maîtrisées » quelles que soient la section et l'activité. Une exception lors de l'accueil des grands s'explique par l'organisation spécifique d'une éducatrice qui oriente les activités des enfants au fur et à mesure de leur arrivée profitant de leur début d'autonomie.
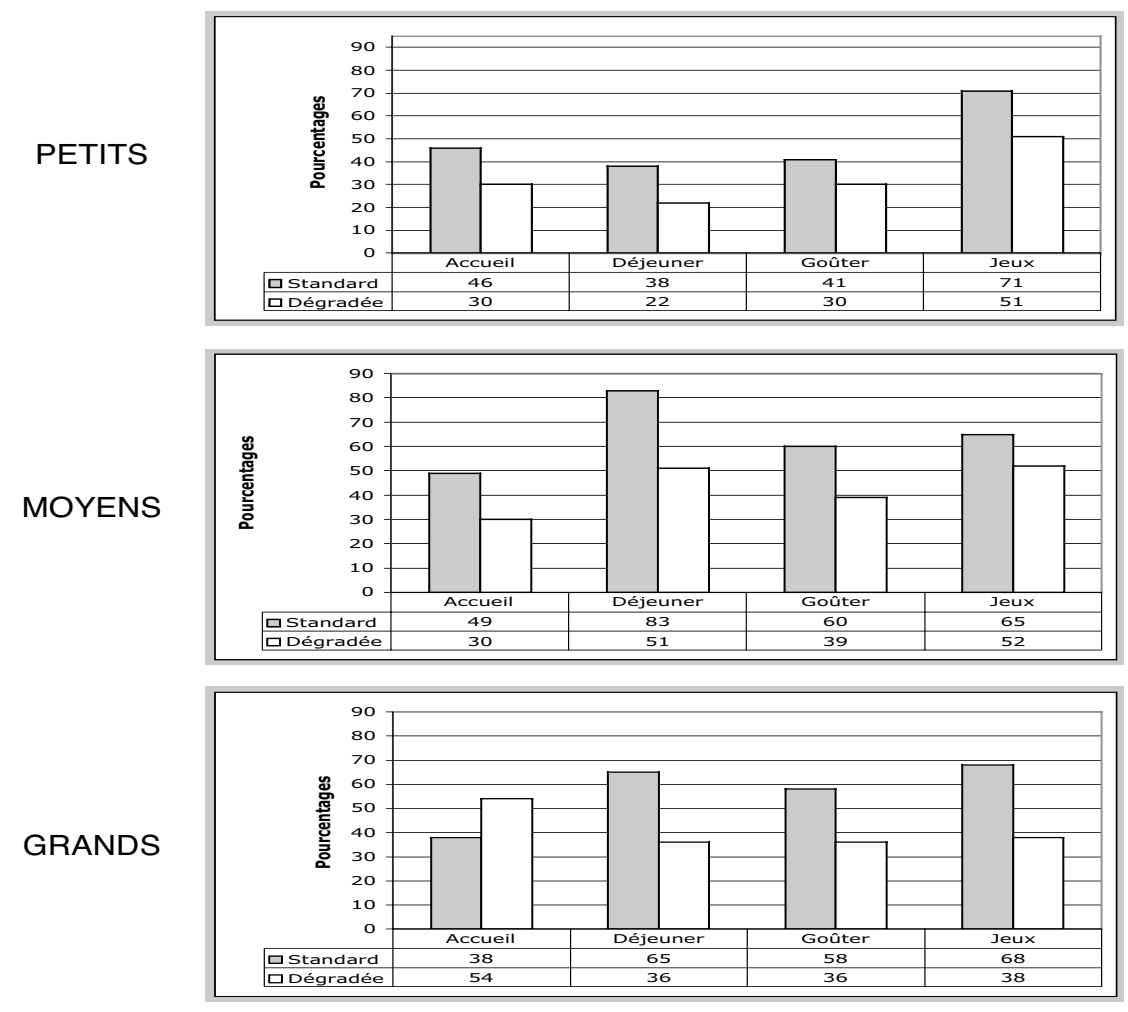

Figure 3.- Pourcentage d'interventions de la catégorie « régulation maitrisée » dans la section des petits, des moyens et des grands, en situations «standard » et« dégradée » 
Les conséquences de la charge de travail en situation dégradée varient donc selon la section et les activités en cours. Cependant, le regroupement des différentes dimensions masque le fait que ce ne sont pas les mêmes caractéristiques et tonalités des relations qui sont en cause dans chaque cas.

Afin de comparer plus finement les modifications du comportement en situation dégradée, un indice de tendance a permis d'estimer l'importance de la variation de chaque tonalité et dimension dans chaque section et pour chaque activité. Sans entrer dans les détails (Gonzales, 2004), le principe de cette évaluation est une estimation des différences entre fréquences attendues sous hypothèse nulle et fréquences observées :

\begin{tabular}{|c|c|c|c|c|c|c|c|}
\hline & \multicolumn{3}{|c|}{ Régulation maîtrisée } & \multicolumn{3}{|c|}{ Régulation opportuniste } \\
\hline & & $\begin{array}{l}\text { Orientations } \\
\text { pédagogique }\end{array}$ & \begin{tabular}{c|} 
Relationnelle \\
Encouragement
\end{tabular} & $\begin{array}{c}\text { Organisation- } \\
\text { nelle proactive }\end{array}$ & $\begin{array}{l}\text { Relationnelle } \\
\text { corrective }\end{array}$ & $\begin{array}{l}\text { Relationelle pro- } \\
\text { tetrice }\end{array}$ & $\begin{array}{l}\text { Organisation- } \\
\text { nelle réactive }\end{array}$ \\
\hline \multirow{4}{*}{ 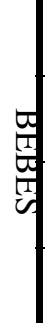 } & Accueil & & 4 & & & 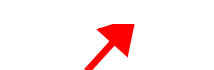 & \\
\hline & Déjeuner & & & & & $\nabla$ & $\nabla$ \\
\hline & Goûter & & & & & 入 & $\nabla$ \\
\hline & Jeux & & & $\lambda$ & & & \\
\hline \multirow{4}{*}{ 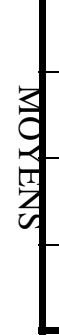 } & Accueil & $\nabla$ & & 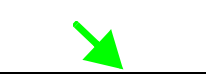 & $\nabla$ & $\nabla$ & $\nabla$ \\
\hline & Déjeuner & $y$ & 1 & 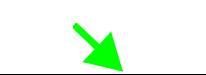 & $\lambda$ & $\nabla$ & $\nabla$ \\
\hline & Goûter & & 1 & 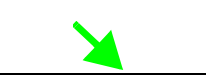 & & & $\gamma$ \\
\hline & Jeux & & & & & & $\nabla$ \\
\hline \multirow{4}{*}{ 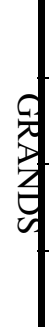 } & Accueil & 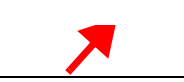 & & & & $\nabla$ & 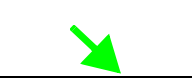 \\
\hline & Déjeuner & & & & $\lambda$ & $\lambda$ & $\nabla$ \\
\hline & Goûter & $M$ & & $M$ & & & $\nabla$ \\
\hline & Jeux & & & & $\nabla$ & & $\nabla$ \\
\hline
\end{tabular}

Tableau 5.- Principales variations de tonalités entre situations standard et dégradée selon les activités dans les différentes sections

Les flèches du tableau 5 indiquent le sens des variations de tonalités les plus importantes entre situation standard et situation dégradée pour les différentes activités dans les trois sections. Les cases vides indiquent une relative stabilité dans la tonalité des interventions. On retiendra :

- Une diminution des interventions dans la «dimension pédagogique » quelle que soit la section sauf en ce qui concerne l'accueil des grands, expliqué précédemment.

- Une diminution de la tonalité « relationnelle - encouragement» dans la section des bébés et des moyens pour les repas, une relative stabilité pour toutes les autres activités et dans la section des grands.

- Une augmentation de la tonalité « relationnelle - corrective » dans les sections des moyens pour l'accueil et le déjeuner ainsi que pour le déjeuner et les jeux des grands. 
- Une augmentation de la tonalité « relationnelle - protectrice » dans la section pour toutes les sections lors de l'accueil et du déjeuner.

- Une diminution de la tonalité " organisationnelle - proactive » pour le déjeuner des moyens et le goûter des grands et une augmentation pour les jeux dans la section de bébés.

- Une augmentation de la tonalité « organisationnelle - réactive » dans toutes les sections et activités sauf l'accueil des grands, ce qui a été expliqué précédemment.

En référant ces variations aux relations qu'elles impliquent avec l'enfant, on constate d'abord une forte tendance à la diminution des relations individuelles dans la « dimension pédagogique » et dans la tonalité « relationnelle - encouragement » ainsi qu'une diminution des activités organisées au préalable («organisationnelle - proactive »). Dans les mêmes séquences d'observation, les tonalités 《 relationnelles - protectrices » et correctives ainsi que la tonalité « organisationnelle - réactive » augmente. Or, par définition, ces tonalités ont pour but d'assurer au coup par coup, la sécurité et la protection des enfants, en réaction à des circonstances jugées risquées. La surveillance et la gestion d'incidents prendraient alors le pas sur les objectifs de développement et de confort et remettraient en cause l'organisation préalable des sections.

Les différences de modalités de régulation observées selon les sections s'expliquent aussi en partie par le développement des capacités des enfants.

- Dans la section des bébés, les demandes et les soins sont très individualisés pour les repas, la propreté, le sommeil ; il y a peu de jeux en commun pour les plus petits mais les progrès moteurs (roulé, quatre pattes et début de la marche) sont rapides entre 3 mois et un an. Laissés pour jouer sur le tapis, les enfants y évoluent un peu à leur fantaisie. On constate alors à la fois une augmentation des tonalités « relationnelle - protectrice » et « organisationnelle - réactive » répondant à des actions risquées (escalades de fauteuil, par exemple), à des disputes avec pleurs et cris pour un jouet, à des maladresses ou à des agressions physiques mais également à une augmentation de la tonalité « organisationnelle - proactive » pour gérer spatialement le groupe. Par exemple, certains bébés sont mis au lit, d'autres regroupés autour de l'auxiliaire, une barrière empêchant les enfants de s'éparpiller.

- Dans la section des moyens, entre 1 et 2 ans, les enfants sont en train d'acquérir de nouvelles capacités motrices et langagières, prennent davantage d'initiatives sans toujours mesurer l'impact de leurs gestes, peuvent être plus exigeants quant à l'attention qu'on leur accorde, plus enclins à «attaquer» les autres si quelque chose leur déplait ou simplement pour avoir le jouet de l'autre par imitation. Il est nécessaire de leur apprendre les règles et de marquer les limites. Ce sont ceux qu'il faut le plus « reprendre » dans la plupart des activités et qui semblent les plus difficiles à contrôler en situation dégradée. Aussi, les variations de tonalités sont-elles les plus importantes dans la plupart des activités, quelle que soit la dimension considérée.

- Dans la section des grands, entre 2 et 3 ans, les enfants commencent à marcher et à parler; ils comprennent et savent obéir à des ordres simples, peuvent jouer ensemble et ont acquis suffisamment d'autonomie pour avoir moins besoin de relations individualisées avec les auxiliaires et se débrouiller seuls dans le cadre d'activités prévues. Ce qui explique la relative stabilité des régulations maîtrisées et que l'augmentation des régulations opportunistes n'atteigne pas l'amplitude que l'on trouve chez les moyens.

Ainsi, en situation dégradée, les caractéristiques des enfants, leur possibilité de contrôle sur leurs propres activités, leur degré d'autonomie se conjuguent avec le type d'activité et le taux d'encadrement pour conduire les auxiliaires à intervenir en priorité sur une surveillance à court terme orientée vers leur protection et leur sécurité, au détriment de relations davantage orientées vers leur progrès et leur confort affectif. Mais il s'agit moins d'un changement de règles que d'une modulation de priorité en réponse à un nombre plus important d'enfants entraînant une agitation plus grande qui interfère avec la gestion prévue d'une activité et peut aller quelquefois jusqu'à l'empêcher. 


\section{Conclusion}

Dans la ligne théorique de « l'agir organisationnel » (Maggi, 2003), l'analyse des modalités de régulation du processus de travail en crèche a permis de mettre en évidence la manière dont les règles de fonctionnement sont posées, réélaborées et modulées tout au long du développement des activités depuis leur conception jusqu'à leur réalisation, en fonction des astreintes ressenties d'un centre de décision à l'autre, selon les niveaux hiérarchiques considérés.

Au niveau institutionnel, macro, la conjonction de règles régissant la durée de travail et les effectifs entraînent, quand elles sont appliquées au niveau managérial, des absences structurelles non remplacées et donc des perturbations dans l'organisation du travail.

$\mathrm{Au}$ niveau meso, les tentatives faites pour réélaborer ces règles et les rendre compatibles avec les horaires effectifs et les buts de l'accueil des enfants impliquent de la part des directrices, dans certains cas des prises d'autonomie par rapport à certaines règles préalables et dans d'autres cas d'agir à leur discrétion. Mais leurs initiatives sont toujours limitées par leur hiérarchie, leur collègues ou les parents qui ont d'autres obligations extérieures à la crèche. Les perturbations ne peuvent donc être compensées que partiellement tant du point de vue organisationnel que temporel. Par suite, les situations standard sont extrêmement rares. Les compromis et les situations dégradées sont ressentis, tant au niveau meso qu'au niveau micro, comme une atteinte au bien-être cognitif et affectif des différents acteurs en provoquant un sentiment de ne pas pouvoir faire face, d'insatisfaction, d'insécurité et d'échec.

Au niveau micro des sections, les situations dégradées entraînent moins des changements de règles que des modifications du comportement des auxiliaires qui doivent faire face aux modifications de comportement des enfants pour tenter de préserver l'équilibre du système d'activités. Cependant, d'un point de vue psychologique, les changements de tonalités dans les différentes dimensions des activités de service vont dans le sens de compensations partielles. L'augmentation des régulations au coup par coup, souvent peu efficaces au détriment de régulations davantage anticipées, traduit les difficultés à mettre en œuvre une organisation de l'action correspondant aux compétences des auxiliaires. Les tensions provoquées par les interruptions de l'activité en cours et les difficultés à faire ce qui est prévu induisent également énervement et fatigue.

$\mathrm{Au}$ final, la qualité de service rendu aux enfants repose sur les «régulations maitrisées ou opportunistes » mises en œuvre par les auxiliaires et les éducatrices, pour gérer en section (petit, moyens et grands), les variations des conditions externes et internes de l'activité et leurs effets. Cependant, la pertinence et l'efficacité de ces régulations par rapport aux enfants, ainsi que la pénibilité des astreintes auxquelles les PPE sont soumises, restent dépendantes de la gestion des relations de service en amont de la crèche. La qualité de service en crèche repose donc sur les articulations des règles et des moyens disponibles dans l'ensemble du système et non uniquement sur les auxiliaires en section.

Sur le plan pratique, les difficultés de gestion des situations mises en évidence dans cette recherche ont permis une concertation entre les crèches et la collectivité locale qui s'est traduite par :

- Le déblocage au Conseil Municipal d'un budget annuel pour assurer l'embauche d'auxiliaires de puériculture pour tous les établissements municipaux de la Ville et la création d'un groupe de PPE volantes pour assurer le remplacement des congés prévisibles.

- L'embauche d'une nouvelle coordinatrice du secteur de la petite enfance dans la commune qui fasse le lien avec les cadres de la Mairie et l'élu de la petite enfance.

- La demande d'un financement auprès de la Caisse d'Allocations Familiales, pour la dotation de matériel informatique pour garantir la gestion du personnel, du matériel et de la communication avec la Mairie. 
On doit ajouter à ces transformations organisationnelles, une satisfaction avouée du personnel de la crèche que la recherche a aidé à prendre conscience de certains aspects de leur métier et à mieux le faire reconnaître à l'extérieur.

Enfin, ces améliorations sur le plan organisationnel montrent aussi en quoi une analyse ergonomique peut contribuer à résoudre un problème apparemment du ressort des ressources humaines.

\section{BIBLIOGRAPHIE}

Baudelot, O., Rayna, S., \& Guibert, L. (2000). La construction des partenaires dans la coordination municipale de la petite enfance. In F. Platone, \& M. Hardy (Eds), On n'enseigne pas tout seul à la crèche, à l'école, au collège, et au lycée (pp. 83-88). Paris: INRP.

Bouve, C. (2001). Les crèches collectives : usagers et représentations sociales. Paris : L'Harmattan.

Caroly, S. (2002). "Gérer les règles et le client" : implication des cadres dans les modalités d'ajustement des règles pour une organisation efficiente. In Actes du 37ème Congrès de la Société d'Ergonomie de Langue Française, Les évolutions de la prescription (pp. 276-284). Aix-en-Provence, France.

Caroly, S., \& Clot, Y. (2004). Du travail collectif au collectif de travail: développer des stratégies d'expérience. Formation et emploi (Numéro spécial « autour de l'expérience et de sa validation », 88, 4355 .

Cristofari, MF., \& Miranda, A. (1988). La socialisation d'un rapport intime à caractère privé. Les métiers de la petite enfance. In Groupe d'études sur la division sociale et sexuelle du travail (Gedisst) (Eds.), La petite enfance : pratiques et politiques (pp. 125-149). Paris: L'Harmattan, Cahiers du genre ${ }^{\circ} 22$.

Dejours, Ch. (2001). Évaluer les activités de service ? Le Monde économie, 13 février.

De la Garza, C. Maggi, B., \& Weill-Fassina, A. (1999). Modalités de réélaboration des règles : des moyens de compensation des perturbations dans la maintenance d'infrastructures ferroviaire. Actes du $34^{\mathrm{ème}}$. Congrès de la SELF, Caen, pp. 335-343.

De Keiser ,V. (1990). Erreurs temporelles et intégration dans les systèmes dynamiques. In J. Leplat, \& G. de Terssac (Eds), Les facteurs humains de la fiabilité dans les systèmes complexes ( pp. 85-108). Marseille: Octarès Éditions.

Donati P., Mollo S., Norvez A., \& Rollet C. (1999). Les centres maternels. Réalité et enjeux éducatifs. Paris: L'Harmattan.

Dubet, F. (2002). Le déclin de l'institution. Paris: Seuil.

Durning, P. (1986). Éducation et suppléance domestique. Psycho-sociologie de l'internat «spécialisé ». Laboratoire de sciences de l'éducation, Université Paris X Nanterre.

Falzon, P. (2000). Ergonomics and customer-operator interactions. In Proceeding of IEA Congress San Diego, Californie, Volume 2, pp. 551-554.

Faverge, J-M. (1979-80). Le travail en tant qu'activité de récupération. Bulletin de Psychologie, XXXIII (344), 203-206.

Faverge, J-M., Olivier, M., Delahaut J., Stephaneck, P., \& Falmagne J.C. (1970). L'ergonomie des processus industriels. Études de psychologie sociale et industrielle. Université Libre de Bruxelles.

Goffman, E. (1968). Asiles. Études sur les conditions sociales des malades mentaux. Paris: Editions de Minuit.

Gonon, O. (2003). Des régulations en lien avec l'âge, la santé et les caractéristiques du travail : le cas des infirmières d'un centre hospitalier français. Pistes, 5(1) http//www.pistes.uqam.ca/v5n1/articles/ v5n1a1.htm

Gonzalez, R. (2004). Du macro au micro : les régulations des relations de service en crèches. Thèse de Doctorat en Ergonomie, Laboratoire d'Ergonomie du CNAM, Paris.

Gonzalez, R., Claire-Louisor, J., \& Weill-Fassina, A. (2001). Les activités psycho-socio-éducatives : une catégorie spécifique de relation de service. Actes du Congrès de la SELF-ACE, 3-5 octobre, Montréal (Canada). Volume 6, pp. 76-82. 
González, R., \& Teiger, C. (2001). Restitution en continu et construction de pistes de transformation au cours d'une recherche en ergonomie. Actes du Congrès de la SELF-ACE, 3-5 octobre, Montréal (Canada), Volume 3, pp. 30-35.

Grosjean, M. (2000). Hétérogénéité des Métiers de Service au Public, hétérogénéité des compétences. 11ème Congrès de psychologie du travail et des organisations, Symposium : Les nouveaux métiers de service au public, Rouen.

Grossin, W. (1996). Pour une science des temps, introduction à l'écologie temporelle. Toulouse: Octarès Éditions.

Hardy, M., Platone, F., \& Stambak, M. (1991). Naissance d'une pédagogie interactive. Paris: INRP.

Hubault, F., \& Bourgeois, F., (2002) La relation de service : une convocation nouvelle pour l'ergonomie. In F. Hubault (Ed.), La relation de service, opportunités et questions nouvelles pour l'ergonomie (pp. 6-31). Toulouse: Octarès Éditions.

Inhelder, B. (1987). Des structures aux processus .In J. Piaget, P. Mounoud, \& J.-P. Bronckart (Eds ?), Psychologie (pp. 654-679). Bourges Gallimard. Encyclopédie de la Pléiade.

Jenson, J., \& Sineau, M. (2001). Who Cares? Women's Work, Childcare and Welfare State Redesign. Toronto: University of Toronto Press.

Letablier, M-T. (2001). Le travail centré sur autrui et sa conceptualisation en Europe. Travail, Genre et Sociétés. La revue du mage. Dossiers : Femmes providentielles, enfants et parents à charge, 6, 20-41.

Maggi, B. (1996). La régulation du processus d'action sociale. In P. Cazamian, F. Hubault, \& M. Noulin (Eds.), Traité d'ergonomie (pp. 637-662). Toulouse: Octarès Éditions.

Maggi, B. (1999). Method of organisationnal congruencies and ergonomic work analysis: characteristics and complementarity. Ergonomia, 12, 44-45.

Maggi, B. (2003). De l'agir organisationnel. Un point de vue sur le travail, le bien-être, l'apprentissage. Toulouse: Octarès Éditions.

Marcos, H., Orvig-Salazar, A., Bernicot, J., Guidette, M., Hudelot, Ch., \& Preneron, Ch. (2000). Le développement du langage et de la communication. L'influence du mode d'accueil chez les enfants de deux et trois ans. Recherches et Prévisions, de la Caisse Nationale des Allocations Familiales, 57-70.

Mauvais, P. (2000). Des formations intra-institutionnelles en pouponnières. Accompagner les équipes dans leur recherche d'un fonctionnement institutionnel centré sur l'enfant. In C.Blanchard-Laville, \& D. Fablet (Eds.), Pratiques d'intervention dans les institutions sociales et éducatives (pp. 253-293). Paris: L'Harmattan.

Meirieu, Ph. (2000). "On n'enseigne pas tout seul", vrai ou faux ? In. F. Platone, \& M. Hardy (Eds.), On n'enseigne pas tout seul, à la crèche, à l'école, au collègue et au lycée (pp. 9-11). Paris: INRP.

Piaget J. (1975). L'équilibration des structures cognitives: problème central du développement. Études d'épistémologie génétique, Volume 33, Paris : PUF.

Pirard F. (1997). Accompagnement pour un accueil de qualité. In S. Rayna, \& F. Dajez (Eds.), Formation petite enfance et partenariat (pp. 151-177). Paris: CRESAS 13.

Pueyo, B. (1999). Le journal de bord, un outil d'observation en formation initiale d'éducateurs de jeunes enfants. In C. Blanchard-Laville, \& D. Fablet (Eds.), Développer l'analyse des pratiques des professionnels de l'intervention socio-éducative (pp. 37-58). Paris: L'Harmattan.

Pueyo V., \& Gaudart C. (2000). L'expérience dans les régulations individuelles et collectives des déficiences. In T.H. Benchekroun, \& A. Weill-Fassina (Eds.), Le Travail Collectif. Perspectives actuelles en ergonomie (pp. 257-272). Toulouse : Octares Éditions.

Robert P. (1994.) Régulation. In J. Rey-Debove, \& A. Rey (Eds.), Le nouveau petit Robert. PARIS: Dictionnaire Le Robert (1911).

Sperandio, J-C. (1972). Charge de travail et régulation des processus opératoires. Le Travail Humain, 35 (1), 85-98.

Stamback, M. (2000). Donner à tous envie d'apprendre. Le défi du Cresas. In F. Platone, \& M. Hardy (Eds.), On n'enseigne pas tout seul, à la crèche, à l'école, au collège et au lycée (pp. 17-25). Paris: INRP. 
Terssac, de G. (1992) Autonomie dans le travail. Paris: PUF.

Valot, C., Weill-Fassina A., Guyot, S., \& Amalberti, R. (1996). Vers un modèle pour l'analyse ergonomique des grands systèmes. In Actes du 30ème congrès de la SELF : Ergonomie et production industrielle, L'homme dans les nouvelles organisations (pp. 272-279). Biarritz, France.

Vérillon, A., Belmont, B., Vial, M., \& Portelli, P. (2000). La collaboration entre enseignants et spécialistes dans l'intégration scolaires des enfants handicapés.. In F. Platone, \& M. Hardy (Eds.), On n'enseigne pas tout seul, à la crèche, à l'école, au collège et au lycée (pp. 97-102). Paris: INRP.

Weill-Fassina, A. (1971-1972). La notion de régulation en Psychologie du travail : définitions et cadres généraux . Bulletin de psychologie, Volume XXV, 10-11 N²98, 546-551.

Weill-Fassina A., \& Caroly, S. (2003). Évolutions des recherches ergonomiques sur les relations de service. In Actes des Journées de Bordeaux: 10 ans de pratiques de l'ergonomie: avons-nous beaucoup changé ?(pp. 105-114). Laboratoire d'Ergonomie des Systèmes Complexes, Université Victor Segalen, Bordeaux.

Weill-Fassina, A., \& Valot, Cl. (1998). Le métier ça va, mais le problème, c'est ce qu'il y a autour. In M.F. Dessaigne, \& I.Gaillard (Eds.), Des évolutions en ergonomie (pp. 75-88). Toulouse: Octarès Éditions.

Zaouche-Gaudron, Ch., Ricaud-Droisy, H., \& Beaumatin, A. (2002). Divergences et convergences des pratiques éducatives. 6e Biennale de l'éducation et de la formation. Paris: INRP.

\section{RÉFÉRENCEMENT}

González, R., \& Weill-Fassina, F. (2005). Modalités de régulation du processus de travail dans les activités de service en crèche. @ ctivités, 2 (2), 2-23, http://www.activites.org/v2n2/gonzales.pdf

\section{RESUMEN \\ Modalidades de regulacion del proceso de trabajo en Guardería}

El objetivo de esta investigación es describir las modalidades y los procesos de decisión y de acción que se producen en los distintos niveles de la organización del trabajo de una guardería. Se mostrará cómo las directoras de guarderías y las auxiliares de preescolar, reelaboran cada una en su nivel, las reglas y normas previamente establecidas por las instancias administrativas locales y nacionales; todas efectuadas con el objeto de mantener la calidad de los servicios prestados a los padres (usuarios) y a los niños (beneficiarios). Los resultados obtenidos; basados en métodos de análisis cualitativos y cuantitativos, individuales y colectivos, evidencian que la calidad de atención en cada sección depende por un lado, de las "tonalidades" de las relaciones entre auxiliares y niños, y por el otro por las regulaciones elaboradas por los principales centros de decisión del sistema.

\section{PALABRAS CLAVE}

Actividades de servicio, Regulaciones, Profesionales de preescolar, Guardería.

\section{RÉSUMÉ}

Le but de cette recherche est de décrire les modalités de régulation du processus de décisions et d'actions qui interviennent à différents niveaux de l'organisation du travail dans les relations de service en crèche. On montrera à la suite de quelles astreintes et de quelles manières les Directrices de crèches et les Auxiliaires de Puériculture réélaborent, chacune à leur niveau, les règles préalables fixées par les instances administratives, pour maintenir la qualité des services rendus aux parents et aux enfants. Les 
résultats se fondent sur plusieurs méthodes d'analyse qualitatives et quantitatives, individuelles et collectives. Ils montrent que la qualité des activités de service en section est caractérisable par les «tonalités 》 des relations entre auxiliaires et enfants, mais que cette qualité dépend largement des régulations effectuées en amont par les principaux centres de décision du système.

MOTS-CLÉS

Activités de service, régulations, personnel de la petite enfance, crèche 